\title{
Association between metabolic syndrome and multiple lesions of intracranial atherothrombotic stroke: a hospital-based study
}

\author{
Kazuhiko Kotani ${ }^{1,2,3^{*}}$, Noriko Satoh-Asahara ${ }^{4}$, Takuya Nakakuki ${ }^{5}$, Hajime Yamakage ${ }^{4}$, Akira Shimatsu ${ }^{4}$ \\ and Tetsuya Tsukahara ${ }^{6^{*}}$
}

\begin{abstract}
Background: With the increasing trend of metabolic syndrome (MetS) and atherothrombotic stroke (which can manifest as stroke lesion multiplicity), studies on the association between MetS and the clinical aspects of atherothrombotic stroke are of great interest. The present study aimed to investigate the association between MetS and multiple atherothrombotic strokes in patients with intracranial atherothrombotic stroke.

Methods: A retrospective study based on medical charts was conducted among patients ( $n=202: 137$ men/65 women) who were symptomatically admitted to the hospital with the first-ever atherothrombotic stroke. For the occurrence of multiple lesions of stroke, odds ratio [OR: $95 \%$ confidence interval (CI)] of MetS or its respective components was calculated using logistic regression models.

Results: Fifty-one percent of the men and $38 \%$ of women with stroke presented multiple regions. MetS was a significant factor that was associated with an increased risk of multiple regions in women [OR 4.3 (95\% Cl 1.4-13.5)], but not in men. According to the components of MetS, dyslipidemia was a significant factor that was positively associated with multiple regions in both men [OR 2.0 (95 \% Cl 1.1-3.7)] and women [OR $3.2(95 \% \mathrm{Cl} 1.1-9.1)$ ].
\end{abstract}

Conclusion: MetS may be pathophysiologically associated with intracranial atherothrombotic stroke multiplicity in women in particular. Future studies are warranted to confirm the findings.

Keywords: Insulin resistance, Obesity, Triglyceride, HDL-cholesterol, Dyslipidemia, Ischemic stroke

\section{Background}

Metabolic syndrome (MetS) consists of metabolic abnormalities, such as obesity, high blood pressure (BP), hyperglycemia and dyslipidemia (high triglyceride (TG)/ high-density lipoprotein cholesterol (HDL-C)) [1, 2]. Met $\mathrm{S}$ is considered to be a risk factor of vascular diseases $[1,2]$. Therefore, the elucidation of the clinical relevance of MetS in vascular medicine is of importance.

Stroke is one of the vascular diseases, and a better understanding of the pathophysiology of stroke is crucial

\footnotetext{
*Correspondence: kazukotani@jichi.ac.jp; ttsukaha@kyotolan.hosp.go.jp ${ }^{1}$ Division of Preventive Medicine, Clinical Research Institute, National Hospital Organization, Kyoto Medical Center, Kyoto, Japan

${ }^{6}$ Department of Neurosurgery, National Hospital Organization, Kyoto

Medical Center, Kyoto, Japan

Full list of author information is available at the end of the article
}

given its frequency and global socio-medical burden $[3,4]$. There has been evidence of a positive association between MetS and the prevalence/morbidity of stroke [5-10]. Of note, intracranial atherothrombotic stroke has gradually increased among ischemic stroke patients with the epidemic of MetS in Japan [11]. In fact, a few studies have reported a positive association between MetS and atherothrombotic stroke [7,12-14]. With the growing concern of studies for atherothrombotic stroke [11], the association between MetS and the clinical aspects of atherothrombotic stroke should be further explored.

Atherothrombosis has a biological characteristic to lead to multi-bed vascular disorders [15]. Carotid atherosclerosis, reflective of generalized atherosclerotic manifestations, is reported to increase the risk of vascular events in relation to MetS [16]. It is of interest to study 
the association between MetS and atherothrombotic stroke legion multiplicity; however, there is a paucity of such data. Therefore, the present study aimed to investigate the association between MetS and multiple atherothrombotic strokes in in-patients who were admitted to the hospital with the first-ever stroke.

\section{Methods}

We conducted a retrospective study through an analysis of medical charts (from 2003 to 2006) of patients who were admitted to our hospital with defined intracranial atherothrombotic stroke. The included patients were limited to those with a diagnosis of the first-ever symptomatic stroke. The diagnosis of atherothrombotic stroke was made according to the diagnostic criteria of the National Institute of Neurological Disorders and Stroke by neurological specialists [17]. That is, it was diagnosed in cases with infarcts greater than $1.5 \mathrm{~cm}$ in diameter of a major brain and/or branch cortical artery on imaging (a computed tomography and/or magnetic resonance imaging) as possible origins under clinical symptoms of neurological deficits [17]. Strokes in patients with a source of embolus, such as atrial fibrillation, moderate-to-severe valvular heart disease or intra-carotid/cardiac thrombus, were excluded as having a definite or possible cardioembolic stroke [17]. Strokes in patients with an undetermined etiology despite an extensive evaluation were also excluded [17]. The study was approved by the Ethics Committee of Kyoto Medical Center.

A total of 202 patients (137 men and 65 women) were enrolled in the study. The clinical data on the components of MetS and stroke multiplicity in the patients was collected based on the description of the medical records. Like the National Cholesterol Education Program Adult Treatment Panel III [1] (the criterion level for obesity and low HDL-C was modified for Japanese people [2, 18]), the patients were diagnosed as having MetS when the patients had at least three components of MetS. The components were obesity (a body mass index $\geq 25 \mathrm{~kg} /$ $\mathrm{m}^{2}$ [2] ), high BP (systolic BP $\geq 130 \mathrm{mmHg}$, diastolic BP $\geq 85 \mathrm{mmHg}$ and/or the use of antihypertensive drugs), hyperglycemia (fasting plasma glucose $\geq 110 \mathrm{mg} / \mathrm{dL}$ and/ or the use of glucose-lowering drugs), high TG (fasting serum triglyceride $\geq 150 \mathrm{mg} / \mathrm{dL}$ and/or the use of triglyceride-lowering drugs) and low HDL-C (fasting serum HDL-C $<40 \mathrm{mg} / \mathrm{dL}$ [18] ). The components of MetS were according to the laboratory data and/or the description in medical records (e.g., if glucose intolerance was clearly described in the charts, it was treated as being within the hyperglycemic criteria).

The differences between the groups were examined by an independent $t$ test or the Chi square test. For the outcome variable (having multiple lesions of stroke), odds ratio [OR: $95 \%$ confidence interval $(\mathrm{CI})$ ] of the explanatory variables (MetS and its components) was calculated using crude and adjusted logistic regression model analyses. In the logistic regression models, high TG and low HDL-C were treated as a dyslipidemic component because few patients had low HDL-C (this is often seen in Japanese people) and the potential collinearity of low HDL-C with high TG (which mirrors the biological nature). The statistical analyses were also performed according to gender. A P value $<0.05$ was considered to be statistically significant.

\section{Results}

Table 1 shows the clinical characteristics of study patients. In men, $51 \%$ of the stroke cases presented multiple regions, while $38 \%$ of women presented multiple regions. There was a significantly higher prevalence of obesity and hyperglycemia in women than in men. In men, the group with multiple legions had a significantly higher prevalence of dyslipidemia (high TG in particular) than the group with a single region. In women, the group with multiple legions had a significantly higher prevalence of dyslipidemia (high TG in particular) and MetS than the group with a single region.

Table 2 shows the OR (95\% CI) of MetS for multiple lesions. Crude and age-adjusted logistic regression model analyses revealed that MetS was a significant factor (approximately 4-fold) that was positively associated with multiple regions in women, but not men.

Table 3 also shows the OR $(95 \% \mathrm{CI})$ of each component of MetS for multiple lesions. The crude and multivariateadjusted logistic regression model analyses revealed that dyslipidemia was a significant factor (approximately 2to 3-fold) that was positively associated with multiple regions in both men and women.

\section{Discussion}

In the present study, MetS showed a significant positive association with multiple lesions of intracranial atherothrombotic stroke in women, but not men, who were in-patients admitted to the hospital with the first-ever stroke. The observation that MetS may be an underlying entity for the formation of intracranial stroke multiplicity with a possible gender bias is new information. Atherothrombotic stroke is considered a treatable target [11], while in patients suffering from multiple lesions of stroke, it may lead to multiple neurological and/or cognitive dysfunctions $[19,20]$. Thus, the treatment of MetS can be significant for preventing atherothrombotic stroke multiplicity and subsequent dysfunctions in women in particular.

A positive association between MetS and atherothrombotic stroke has been reported [7, 12-14], and 
Table 1 Clinical characteristics of the patients

\begin{tabular}{|c|c|c|c|c|c|c|c|c|c|}
\hline & \multicolumn{3}{|l|}{ Men } & \multirow[t]{2}{*}{$\mathbf{P 1}$} & \multicolumn{3}{|l|}{ Women } & \multirow[t]{2}{*}{$\mathrm{P} 2$} & \multirow[t]{2}{*}{ P3 } \\
\hline & All & Single & Multiple & & All & Single & Multiple & & \\
\hline Number, n & 137 (100\%) & 67 (49\%) & 70 (51 \%) & - & 65 (100\%) & $40(62 \%)$ & 25 (38 \%) & - & 0.09 \\
\hline Age (years) & $68 \pm 9$ & $67 \pm 11$ & $69 \pm 7$ & 0.17 & $70 \pm 10$ & $70 \pm 12$ & $69 \pm 7$ & 0.62 & 0.20 \\
\hline Obesity & $28(20 \%)$ & $14(21 \%)$ & $14(20 \%)$ & 0.90 & $24(37 \%)$ & $12(30 \%)$ & $12(48 \%)$ & 0.14 & $0.01^{*}$ \\
\hline High BP & $101(74 \%)$ & $49(73 \%)$ & $52(74 \%)$ & 0.88 & $41(63 \%)$ & $24(60 \%)$ & $17(68 \%)$ & 0.52 & 0.12 \\
\hline Hyperglycemia & $64(47 \%)$ & $26(39 \%)$ & $38(54 \%)$ & 0.07 & $42(65 \%)$ & $23(58 \%)$ & $19(76 \%)$ & 0.13 & $0.02^{*}$ \\
\hline High TG/low HDL-C & $70(51 \%)$ & $28(42 \%)$ & $42(60 \%)$ & $0.03^{*}$ & $33(51 \%)$ & $16(40 \%)$ & $17(68 \%)$ & $0.03^{*}$ & 0.97 \\
\hline High TG & $66(48 \%)$ & $26(39 \%)$ & $40(57 \%)$ & $0.03^{*}$ & $31(48 \%)$ & 14 (35 \%) & 17 (68 \%) & $0.01^{*}$ & 0.94 \\
\hline Low HDL-C & $7(5 \%)$ & $3(4 \%)$ & $4(6 \%)$ & 0.74 & $7(8 \%)$ & $3(8 \%)$ & $4(16 \%)$ & 0.28 & 0.14 \\
\hline MetS & 25 (18\%) & $10(15 \%)$ & 15 (21\%) & 0.33 & 19 (29\%) & 7 (18\%) & $12(48 \%)$ & $0.01^{*}$ & 0.08 \\
\hline
\end{tabular}

Independent $t$-test, $\mathrm{P} 1$ : single vs. multiple in men, P2: single vs. multiple in women, P3: all men vs. all women.

$B P$ blood pressure, $T G$ triglyceride, HDL high-density lipoprotein, MetS metabolic syndrome.

Table 2 Odds ratio (95\% confidence interval) of MetS for multiple lesions

\begin{tabular}{lccc}
\hline Variable & All & Men & Women \\
\hline Crude & $2.1(1.1-4.2$, & $1.6(0.6-3.8$, & $4.3(1.4-13.5$, \\
& $\left.p=0.03^{*}\right)$ & $p=0.32)$ & $\left.p=0.01^{*}\right)$ \\
Age-adjusted & $2.2(1.1-4.3$, & $1.7(0.7-4.2$, & $\begin{array}{c}4.3(1.4-13.4, \\
\end{array}$ \\
& $\left.p=0.03^{*}\right)$ & $p=0.24)$ & $\left.p=0.01^{*}\right)$ \\
\hline
\end{tabular}

the etiopathological mechanisms of atherothrombosis (e.g., via vasomotor reactivity impairment, insulin resistance, inflammation, oxidative stress, platelet activation and hypercoagulation) have been proposed [15, 20-24]. Under the etiopathology, atherothrombotic stroke may suddenly occur in multiple lesions, and/or the repeated stroke formation may be unnoticed in some cases whose lesions are multiple, like a silent brain infarct $[25,26]$.
Gender differences exist in the clinical features of MetS, stroke and their association thereof [11, 27-29]; that is, the influences of MetS on stroke may be greater in Japanese women than in men $[27,28]$. According to these literature findings, the associations among MetS and atherothrombotic stroke multiplicity observed in the present survey appear to be plausible. On the other hand, though the impact of MetS on multiple lesions in men was not significant and weaker than that in women, the OR level was over 1.5 (Table 2) and confirmation may be repeatedly required in men. Mutifaceted studies including etiopathological factors and larger samples in both genders will be necessary to understand the findings.

The clinical significance of MetS or each component of MetS on the atherosclerotic outcomes is poorly understood [30-32]. In the present study, the analysis of each component of MetS with multiple lesions showed

Table 3 Odds ratio (95\% confidence interval) of each component of MetS for multiple lesions

\begin{tabular}{llll}
\hline Variable & All & Men & Women \\
\hline Crude & & & \\
$\quad$ Age (years) & $1.0(1.0-1.0, p=0.52)$ & $1.0(1.0-1.1, p=0.14)$ & $1.0(0.9-1.0, p=0.62)$ \\
Obesity & $1.2(0.6-2.2, p=0.62)$ & $0.9(0.4-1.8, p=0.77)$ & $2.2(0.8-6.1, p=0.15)$ \\
High blood pressure & $1.2(0.7-2.3, p=0.49)$ & $1.4(0.7-2.8, p=0.31)$ & $1.4(0.5-4.1, p=0.52)$ \\
Hyperglycemia & $1.8\left(1.0-3.1, p=0.04^{*}\right)$ & $1.5(0.8-2.8, p=0.16)$ & $2.3(0.8-7.1, p=0.13)$ \\
High TG/low HDL-C & $2.3\left(1.3-4.1, p<0.01^{*}\right)$ & $2.0\left(1.1-3.7, p=0.02^{*}\right)$ & $3.2\left(1.1-9.1, p=0.03^{*}\right)$ \\
Multivariate-adjusted & & & $1.0(0.9-1.0, p=0.56)$ \\
Age, years & $1.0(1.0-1.0, p=0.33)$ & $1.0(1.0-1.1, p=0.05)$ & $1.9(0.6-5.8, p=0.27)$ \\
Obesity & $1.1(0.6-2.2, p=0.69)$ & $1.0(0.4-2.3, p=0.95)$ & $1.4(0.5-4.3, p=0.56)$ \\
High blood pressure & $1.2(0.6-2.3, p=0.54)$ & $1.0(0.4-2.2, p=0.99)$ & $2.2(0.7-7.5, p=0.20)$ \\
Hyperglycemia & $1.7(0.9-3.1, p=0.06)$ & $2.6\left(1.2-5.4, p=0.01^{*}\right)$ & $3.0\left(1.0-8.9, p=0.046^{*}\right)$ \\
High TG/low HDL-C & $2.5\left(1.4-4.4, p<0.01^{*}\right)$ &
\end{tabular}

Multivariate-adjusted model: age, obesity, high blood pressure, hyperglycemia and high TG/low HDL-C were entered into the model as variables for multiple lesions.

MetS metabolic syndrome, TG triglyceride, $H D L$ high-density lipoprotein. 
that dyslipidemia in particular (such as high TG and/or low HDL-C [33]) was positively associated with multiple lesions in both genders. Such a significant influence of dyslipidemia on stroke can be supported by previous studies [11,34], although these studies did not examine stroke multiplicity. In addition to the treatment of MetS, a consideration of atherogenic dyslipidemia [33] could be particularly pivotal when subjects do not necessarily manifest a complete MetS phenotype.

There are several limitations associated with this study. The study had a retrospective design based on clinical data obtained from medical records. Although the multiple lesions detected in the present study could include old infarcts, the clear discrimination between new and old infarcts was not always made (it was difficult to discriminate them based on a retrospective description of medical records). The number of multiple lesions may also be useful information; however, it could not completely be collected in this survey. The additional information (e.g., serum low-density lipoprotein cholesterol, smoking and alcohol intake) was not fully collected for all patients. Information bias may therefore affect the study results. The study was conducted in a single hospital, which could lead to selection bias. Furthermore, there may be ethnic disparities in the relevance of MetS on stroke multiplicity [10] and the definition of MetS itself may affect the results [14, 35]. These issues should be addressed in future studies with prospective and multicentric designs with various populations.

In summary, MetS may have a pathophysiology associated with intracranial atherothrombotic stroke multiplicity in women in particular. Future studies are warranted to confirm the findings.

\section{Authors' contributions}

NS-A and TN made a data set. KK, TN and HY analyzed the data. KK and TN searched the literature. All authors participated in the development of the study and the interpretation of the data. All authors read and approved the final manuscript.

\footnotetext{
Author details

1 Division of Preventive Medicine, Clinical Research Institute, National Hospital Organization, Kyoto Medical Center, Kyoto, Japan. ${ }^{2}$ Division of Community and Family Medicine, Jichi Medical University, Shimotsuke, Japan. ${ }^{3}$ Department of Clinical Laboratory Medicine, Jichi Medical University, Shimotsuke, Japan. ${ }^{4}$ Division of Diabetic Research, Clinical Research Institute, National Hospital Organization, Kyoto Medical Center, Kyoto, Japan. ${ }^{5}$ Department of Neurosurgery, Hikone Municipal Hospital, Hikone, Japan. ${ }^{6}$ Department of Neurosurgery, National Hospital Organization, Kyoto Medical Center, Kyoto, Japan.
}

\section{Acknowledgements}

We thank Kazuya Muranaka and Michiyo Kawahara for their excellent technical assistance.

\section{Compliance with ethical guidelines}

\section{Competing interests}

The authors declare that they have no competing interests.

\section{Funding}

This work was supported in part by Grants from the National Hospital Organization for collaborative clinical research (T.T. and N. S-A.); a Grant-in-Aid for Scientific Research from the Ministry of Education, Culture, Sports, Science, and Technology of Japan (K.K. and N. S-A.); the Ministry of Health, Labour, and Welfare of Japan; the Smoking Research Foundation, and the Research Award of Japanese Society of Anti-Aging Medicine (N. S-A.).

Received: 12 May 2015 Accepted: 4 August 2015

Published online: 14 August 2015

\section{References}

1. Expert Panel on Detection (2001) Evaluation, and treatment of high blood cholesterol in adults. Executive summary of the third report of the National Cholesterol Education Program (NCEP) Expert Panel on Detection, evaluation, and treatment of high blood cholesterol in adults (Adult Treatment Panel III). JAMA 285(19):2486-2497

2. Examination Committee of Criteria for 'Obesity Disease' in Japan (2002) Japan Society for the Study of Obesity. New criteria for 'obesity disease' in Japan. Circ J 66(11):987-992

3. Norrving B, Kissela B (2013) The global burden of stroke and need for a continuum of care. Neurology 80(3 Suppl 2):S5-S12

4. Mehndiratta MM, Khan M, Mehndiratta P, Wasay M (2014) Stroke in Asia: geographical variations and temporal trends. J Neurol Neurosurg Psychiatry 85(12):1308-1312

5. Bang OY (2006) Intracranial atherosclerotic stroke: specific focus on the metabolic syndrome and inflammation. Curr Atheroscler Rep 8(4):330-336

6. Galassi A, Reynolds K, He J (2006) Metabolic syndrome and risk of cardiovascular disease: a meta-analysis. Am J Med 119(10):812-819

7. Li W, Ma D, Liu M, Liu H, Feng S, Hao Z et al (2008) Association between metabolic syndrome and risk of stroke: a meta-analysis of cohort studies. Cerebrovasc Dis 25(6):539-547

8. Mottillo S, Filion KB, Genest J, Joseph L, Pilote L, Poirier P et al (2010) The metabolic syndrome and cardiovascular risk a systematic review and meta-analysis. J Am Coll Cardiol 56(14):1113-1132

9. Li M, LiY, Liu J (2013) Metabolic syndrome with hyperglycemia and the risk of ischemic stroke. Yonsei Med J 54(2):283-287

10. Osborn MF, Miller CC, Badr A, Zhang J (2014) Metabolic syndrome associated with ischemic stroke among the Mexican Hispanic population in the El Paso/US-Mexico border region. J Stroke Cerebrovasc Dis 23(6):1477-1484

11. Uchiyama S (2005) Impact of metabolic syndrome as a risk factor for atherothrombotic stroke. Intern Med 44(10):1021-1022

12. Bang OY, Kim JW, Lee JH, Lee MA, Lee PH, Joo IS et al (2005) Association of the metabolic syndrome with intracranial atherosclerotic stroke. Neurology 65(2):296-298

13. Ovbiagele B, Saver JL, Lynn MJ, Chimowitz M, WASID Study Group (2006) Impact of metabolic syndrome on prognosis of symptomatic intracranial atherostenosis. Neurology 66(9):1344-1349

14. Hata J, Doi Y, Ninomiya T, Tanizaki Y, Yonemoto K, Fukuhara M et al (2010) The effect of metabolic syndrome defined by various criteria on the development of ischemic stroke subtypes in a general Japanese population. Atherosclerosis 210(1):249-255

15. Duvall WL, Vorchheimer DA (2004) Multi-bed vascular disease and atherothrombosis: scope of the problem. J Thromb Thrombolysis 17(1):51-61

16. Novo S, Peritore A, Trovato RL, Guarneri FP, Di Lisi D, Muratori l et al (2013) Preclinical atherosclerosis and metabolic syndrome increase cardio- and cerebrovascular events rate: a 20-year follow up. Cardiovasc Diabetol $12: 155$

17. Adams HP Jr, Bendixen BH, Kappelle LJ, Biller J, Love BB, Gordon DL et al (1993) Classification of subtype of acute ischemic stroke. Definitions for use in a multicenter clinical trial. TOAST. Trial of Org 10172 in Acute Stroke Treatment. Stroke 24(1):35-41

18. Teramoto T, Sasaki J, Ueshima H, Egusa G, Kinoshita M, Shimamoto K et al (2007) Diagnostic criteria for dyslipidemia. Executive summary of Japan Atherosclerosis Society (JAS) guideline for diagnosis and prevention of atherosclerotic cardiovascular diseases for Japanese. J Atheroscler Thromb 14(4):155-158 
19. Román GC (2002) Vascular dementia revisited: diagnosis, pathogenesis, treatment, andprevention. Med Clin North Am 86(3):477-499

20. Yates KF, Sweat V, Yau PL, Turchiano MM, Convit A (2012) Impact of metabolic syndrome on cognition and brain: a selected review of the literature. Arterioscler Thromb Vasc Biol 32(9):2060-2067

21. Arenillas JF, Moro MA, Dávalos A (2007) The metabolic syndrome and stroke: potential treatment approaches. Stroke 38(7):2196-2203

22. Farooqui AA, Farooqui T, Panza F, Frisardi V (2012) Metabolic syndrome as a risk factor for neurological disorders. Cell Mol Life Sci 69(5):741-762

23. Rodríguez-Flores M, García-García E, Cano-Nigenda CV, Cantú-Brito C (2014) Relationship of obesity and insulin resistance with the cerebrovascular reactivity: a case control study. Cardiovasc Diabetol 13:2

24. van Rooy MJ, Pretorius E (2015) Metabolic syndrome, platelet activation and the development of transient ischemic attack or thromboembolic stroke. Thromb Res 135(3):434-442

25. Bokura H, Yamaguchi S, lijima K, Nagai A, Oguro H (2008) Metabolic syndrome is associated with silent ischemic brain lesions. Stroke 39(5):1607-1609

26. Park JH, Kwon HM (2008) Association between metabolic syndrome and previous ischemic lesions in patients with intracranial atherosclerotic stroke. Clin Neurol Neurosurg 110(3):215-221

27. Mak KH, Ma S, Heng D, Tan CE, Tai ES, Topol EJ et al (2007) Impact of sex, metabolic syndrome, and diabetes mellitus on cardiovascular events. Am J Cardiol 100(2):227-233

28. Takahashi K, Bokura H, Kobayashi S, lijima K, Nagai A, Yamaguchi S (2007) Metabolic syndrome increases the risk of ischemic stroke in women. Intern Med 46(10):643-648
29. Niwa Y, Ishikawa S, Gotoh T, Kayaba K, Nakamura Y, Kajii E (2010) Association between stroke and metabolic syndrome in a Japanese population: Jichi Medical School (JMS) Cohort Study. J Epidemiol 20(1):62-69

30. Okada K (2008) Which diagnostic criteria of metabolic syndrome can better predict the risk of ischemic heart disease and stroke in Japanese and Asian populations? Hypertens Res 31(12):2103-2104

31. Kurth T, Logroscino $G$ (2008) The metabolic syndrome: more than the sum of its components? Stroke 39(4):1068-1069

32. Jeremy RW (2011) Metabolic syndrome-is the whole really greater than the sum of the parts? Heart Lung Circ 20(4):211-213

33. Tanne D, Koren-Morag N, Graff E, Goldbourt U (2001) Blood lipids and first-ever ischemic stroke/transient ischemic attack in the Bezafibrate Infarction Prevention (BIP) Registry: high triglycerides constitute an independent risk factor. Circulation 104(24):2892-2897

34. Sone H, Mizuno S, Fujii H, Yoshimura Y, Yamasaki Y, Ishibashi S et al (2005) Is the diagnosis of metabolic syndrome useful for predicting cardiovascular disease in asian diabetic patients? Analysis from the Japan Diabetes Complications Study. Diabetes Care 28(6):1463-1471

35. Milionis HJ, Kostapanos MS, Liberopoulos EN, Goudevenos J, Athyros VG, Mikhailidis DP et al (2007) Different definitions of the metabolic syndrome and risk of first-ever acute ischaemic non-embolic stroke in elderly subjects. Int J Clin Pract 61(4):545-551

\section{Submit your next manuscript to BioMed Central and take full advantage of:}

- Convenient online submission

- Thorough peer review

- No space constraints or color figure charges

- Immediate publication on acceptance

- Inclusion in PubMed, CAS, Scopus and Google Scholar

- Research which is freely available for redistribution

Submit your manuscript at

www.biomedcentral.com/submit

() BioMed Central 